\title{
A current perspective on post-micturition dribble in males
}

\author{
Dae Yul Yang ${ }^{1}$, Won Ki Lee ${ }^{2}$ \\ 'Department of Urology, Kangdong Sacred Heart Hospital, Hallym University College of Medicine, Seoul, '2 Department of Urology, Chuncheon Sacred Heart Hospital, \\ Hallym University College of Medicine, Chuncheon, Korea
}

Post-micturition dribble (PMD) is the involuntary loss of urine immediately after urination. It is classified as a post-micturition symptom and is more common in males. Traditionally, PMD has been considered to be part of the aging process, to have a low prevalence, and to be less bothersome than other lower urinary tract symptoms (LUTS). However, recent evidence suggests that PMD is not less common and does not elicit less discomfort compared with other LUTS in males. Also, PMD seems to commonly overlap with other LUTS and to be associated with erectile dysfunction. Although the etiology of PMD is not fully understood, a weakness or failure of the pelvic floor muscles is considered to be the most important factor. Although bulbar urethral massage and pelvic floor exercises are known to be effective in treating PMD, pharmacologic treatment has not yet been introduced. Recently, the possibility of treating PMD with phosphodiesterase- 5 inhibitor has been suggested.

Keywords: Erectile dysfunction; Lower urinary tract symptoms; Men; Phosphodiesterase 5 Inhibitors; Post-micturition dribble

This is an Open Access article distributed under the terms of the Creative Commons Attribution Non-Commercial License (http://creativecommons.org/licenses/by-nc/4.0) which permits unrestricted non-commercial use, distribution, and reproduction in any medium, provided the original work is properly cited.

\section{INTRODUCTION}

Post-micturition dribble (PMD) is classified as a postmicturition symptom and is more common in males. It has traditionally been considered to be a part of the aging process, to have a low prevalence, and to be less bothersome than other lower urinary tract symptoms (LUTS) [1-4]. Furthermore, available diagnostic tools and treatment options for PMD are lacking [5-8]. For these reasons, PMD seems to be outside of physicians' and researchers' interests.

In contrast with what is traditionally believed, however, recent studies suggest that PMD may be one of the most frequent and bothersome LUTS in males [9-13]. Also, it has recently been suggested that phosphodiesterase-5 inhibitors
(PDE-5Is) may have a role in relieving PMD [5,8]. This evidence makes it necessary to review PMD. Accordingly, in this review, we provide a current perspective on PMD in males with respect to epidemiology, pathophysiology, and treatment.

\section{EPIDEMIOLOGY}

\section{Definition}

The term $P M D$ is used to describe the involuntary loss of urine immediately after an individual finishes passing urine, usually after leaving the toilet in males or after rising from the toilet in females. It is classified as a post-micturition symptom, according to the standardization of terminology of

Received: 1 April, 2019 - Accepted: 19 April, 2019

Corresponding Author: Won Ki Lee

Department of Urology, Chuncheon Sacred Heart Hospital, Hallym University College of Medicine, 77 Sakju-ro, Chuncheon 24253, Korea

TEL: +82-33-240-5161, FAX: +82-33-240-5426, E-mail: rheewk@hanmail.net

ORCID: https://orcid.org/0000-0001-7172-0636

(C) The Korean Urological Association

www.icurology.org 
LUTS by the International Continence Society [14].

PMD is completely distinguishable from terminal dribble. Terminal dribble is classified as a voiding symptom and is the term used when an individual describes a prolonged final part of micturition when the flow has slowed to a trickle or dribble [14].

The International Prostate Symptom Score (IPSS) is the most widely used tool for the evaluation of LUTS. However, PMD cannot be assessed by using the IPSS, because there are no questions concerning PMD on the IPSS. Therefore, most studies have used the Danish Prostatic Symptom Score (DAN-PSS-1) or questionnaires developed by researchers to assess PMD [15]. However, the DAN-PSS-1 questionnaire does not include information on the frequency of PMD or its effect on quality of life, although the severity of symptoms and associated bother can be assessed. Recently, the Hallym Post-Micturition Dribble Questionnaire (HPMDQ) was introduced to assess PMD (Supplementary material) [5,8]. It was designed by Yang et al. [5] to allow for the assessment of various aspects of PMD, including frequency, severity, bother, quality of life, and response to treatment. However, the HPMDQ has not yet been validated, and further studies are needed to prove its clinical utility.

\section{Prevalence}

In a population-based study (EPIC study) that involved more than 8,000 males aged $\geq 18$ years in five Western countries, the prevalence rate of PMD was 5.5\% [4]. Another population-based study (Boston Area Community Health [BACH] study) involving more than 2,300 males aged 30 to 79 years in the United States reported a prevalence rate of $8.7 \%$ [9]. In a Chinese population study, which involved more than 1,500 males aged $\geq 18$ years, the prevalence rate was $9.4 \%$ [16]. On the other hand, the populationbased Tampere Ageing Male Urologic Study (TAMUS), which involved over 7,000 Finnish males aged 30 to 80 years, revealed a PMD prevalence rate of $58.1 \%$ [10]. The internet-based epidemiologic study (Epidemiology of LUTS, EpiLUTS), which involved over 14,000 males $\geq 18$ years in three Western countries, reported a prevalence rate of $29.7 \%$ [11]. In a practice-based study that involved more than 1,500 males aged $\geq 18$ years in Southeast Asia, the prevalence rate was $55.0 \%$ [17]. In most studies, a positive trend was observed between PMD prevalence and advancing age [4,9-11,16].

In previous studies, the difference in reported prevalence rates seems to be due to the different definition of PMD and the use of various tools for assessing PMD. For example, some studies used the DAN-PSS-1, whereas other studies used questionnaires developed by the researchers. However, it is noted that PMD is not a rare symptom compared with other LUTS. Furthermore, PMD may be one of the most common LUTS in males.

\section{Frequency and amount}

Little information is available concerning the frequency and amount of PMD. In a study that involved 138 males aged 20 to 70 years with PMD and other LUTS, 42.8\%, 32.6\%, and $24.6 \%$ of patients experienced PMD 1 of 3 times, 2 of 3 times, and almost always, respectively [8]. Yang et al. [5] assessed the frequency and amount of PMD in 205 males aged $\geq 40$ years with LUTS. In males without ED, $65.7 \%$, $22.9 \%$, and $11.4 \%$ of patients experienced PMD 1 of 3 times, 2 of 3 times, and almost always, respectively. On the other hand, in males with $\mathrm{ED}, 43.2 \%, 33.0 \%$, and $23.9 \%$ of patients experienced PMD, respectively. The amount of PMD in males without ED was approximately $1.2 \mathrm{~mL}$, whereas it was approximately $2.1 \mathrm{~mL}$ in males with $\mathrm{ED}$.

\section{Quality of life}

In a population-based study that involved 1,709 males aged 18 to 79 years in Finland, the most prevalent LUTS causing moderate or severe bother was PMD [12] However, from an individual perspective, urge incontinence was the most bothersome symptom. In the TAMUS, PMD was the most bothersome LUTS in males aged 30 to 40 years, but not in other age groups [13]. The BACH study showed that post-micturition symptoms including PMD were indicative of impaired physical and mental health-related quality of life using the Medical Outcomes Study 12-item Short Form Survey (SF-12) [9]. In the Chinese population study, PMD was one of the most bothersome LUTS [16]. A practice-based study performed in Southeast Asia reported that more than half of males felt at least some degree of bother for PMD, similar to other LUTS [17].

In previous studies, the degrees of bother for PMD varied. These results were likely affected by the various tools used to assess the degree of PMD. Also, the results might be affected by whether the study was performed at the population level or at the individual level. At the population level, the degree of bother strongly depends on the frequency of PMD. Meanwhile, at the individual level, the variation in level of bother might determine the most bothersome LUTS. However, like PMD prevalence, it is obvious that PMD does not elicit less discomfort than do other LUTS. Furthermore, it may be one of the most bothersome of the LUTS in males.

\section{Association with other LUTS}

In the BACH study, $53.4 \%$ of males with post-micturition 
symptoms had PMD alone, and 21\% had both PMD and incomplete emptying [9]. A total of $24.8 \%$ of males with postmicturition symptoms had no other LUTS. Males with postmicturition symptoms most of ten had co-occurring frequency (approximately 60\%) and more often had co-occurring storage symptoms than voiding symptoms. Conversely, cooccurring post-micturition symptoms were most often seen in males with intermittency (more than 60\%) and were more often reported in males with voiding symptoms than in males with storage symptoms. In a practice-based study performed in Southeast Asia, 1.4\% of males with any LUTS had only PMD alone [17]. Males with PMD had co-occurring weak stream (47.1\%), nocturia (46.4\%), and urgency (35.5\%). Kobayashi et al. [18] assessed the association between PMD and prostate volume and urinary flow in 172 Japanese males aged 40 to 79 years. They reported that there was no relationship between the presence of PMD and prostate size or abnormal urinary flow. On the other hand, Lee et al. [19] assessed 309 males aged $\geq 40$ years with LUTS and reported that PMD frequency was associated with prostate size but not urinary flow.

Studies on the association between PMD and other LUTS are lacking. However, PMD seems to commonly overlap with other LUTS, thereby causing further aggravation.

\section{Association with erectile dysfunction}

Few studies have specifically assessed the association between PMD and erectile function, although it is well known that LUTS are closely associated with erectile dysfunction (ED). In a practice-based study that involved 1,271 males aged $\geq 45$ years in 12 countries, PMD was associated with reduced rigidity of erections (odds ratio [OR], 1.68), reduced ejaculation (OR, 179), and pain on ejaculation (OR, 1.66) [20]. However, in the EpiLUTS, PMD was not associated with sexual activity or ED [21]. Recently, Yang et al. [5] assessed the correlation between PMD and ED in 205 Korean males with LUTS aged $\geq 40$ years. PMD was assessed by using a self-administered questionnaire developed by the authors and by using PMD volume. In that study, ED was well correlated with PMD frequency $(r=0.388)$ and volume $(r=0.138)$. When compared with IPSS measures one-on-one, the $r$ for the correlation of PMD frequency with ED was not lower than the correlation for each of the other IPSS measures, but rather was higher than that for some of the other measures (e.g., incomplete emptying and weak stream).

The mechanism through which PMD might be correlated with ED remains unknown. One hypothesis is a weakened urethro-corporocavernosal reflex [5]. During urination, cavernosa muscle contraction and sinusoidal corpora mus- cle relaxation are suggested to be mediated through the urethro-corporocavernosal reflex [22]. These changes are likely to cause a mild penile tumescence, which can assist in urinary flow and may allow urine to be easily discharged, leading to the prevention of PMD [22-24]. The changes in the anatomical structure of the corpus cavernosum, including the decreased smooth muscle components and enlarged vascular lumens, limit the basic function of the penis, leading to a weakened urethro-corporocavernosal reflex as well as ED [25,26]. A weakened urethro-corporocavernosal reflex could interfere with urinary flow in the urethra during urination, leading to PMD. Pelvic floor muscles are another factor that may explain the correlation between PMD and ED. Pelvic floor muscles are considered to be the most important factor for developing PMD [27]. They also play an active role during sexual activity [1].

The association between PMD and ED is not yet clear. However, considering the close association between storage and voiding LUTS and ED, PMD and ED are likely to be associated.

\section{PATHOPHYSIOLOGY}

For whatever reason, PMD occurs due to urine residue in the bulbar or prostatic urethra after urination [7] However, the pathophysiology by which urine residue remains is not fully understood.

One putative mechanism is a weakness or failure of the pelvic floor muscles, especially the bulbocavernosus and ischiocavernosus muscles [27]. Contraction of the pelvic floor muscles empties the residual urine out of the urethra at the end of urination (called milk-out). Therefore, weakness or failure of the pelvic floor muscles results in poor milk-out, leading to urine residue. Wille et al. [28] performed voiding cystourethrogram (VCUG) before and after radical prostatectomy to assess whether PMD is associated with altered post-voiding milk-out. Before surgery, no patients with normal milk-out had PMD, whereas $86 \%$ of patients without normal milk-out had PMD. After surgery, $87 \%$ of patients with abnormal milk-out reported PMD. They postulated that the bulbocavernosus and ischiocavernosus muscles were involved in the milk-out mechanism at the end of urination. Similarly, Bader et al. [29] also performed VCUG before and after radical prostatectomy to determine post-voiding milkout. They suggested that PMD was associated with the loss of milk-out.

Another putative mechanism is an incompetent external urethral sphincter [27]. At the end of urination, the external urethral sphincter contracts. The urine that remains in the 
prostate urethra is then drawn back into the bladder (called milk-back). An incompetent external urethral sphincter may result in poor milk-back. Immediately after urination, urine trapped in the prostate urethra could fall into the bulbar urethra, leading to urine residue. A similar mechanism can be applied to bladder neck obstruction. Bladder neck obstruction can interfere with milk-back at the end of urination. When the external urethral sphincter relaxes, urine trapped in the prostate urethra could fall into the bulbar urethra, leading to urine residue.

\section{TREATMENTS}

Bulbar urethral massage and pelvic floor exercise are known to relieve PMD. Both methods can be used alone or in combination with each other. Considering that a weakness or failure of pelvic floor muscles might cause PMD, it seems plausible that these two treatment methods have been used to relieve PMD [27]. However, few studies have proven the effectiveness of these methods.

Bulbar urethral massage immediately after urination is a self-help technique and is the simplest way to relieve PMD [30]. The patient is taught to place his fingers behind his scrotum immediately after urination and to gently massage his bulbar urethra in a forwards and upwards direction towards the penis. It may be necessary to repeat this technique several times, until the patient is sure that no urine residue remains [7,31,32]. Similarly, stretching, squeezing, or shaking the penis can help to empty residual urine out of the urethra [32].

Pelvic floor exercise is specifically targeted to the bulbocavernosus and ischiocavernosus muscles [7,32] It can help to place pressure on the bulbar urethra and force out the urine residue. The effect of pelvic floor exercise depends on the weakness of the pelvic floor muscles. Pelvic floor exercise generally does not show an immediate effect; it may take 3 to 6 months to show an adequate effect [33]. It is also difficult to learn and maintain pelvic floor exercise without proper supervision. Paterson et al. [34] compared the effects of counseling, bulbar urethral massage, and pelvic floor exercise on PMD. A total of 49 males with PMD were randomized and followed the treatment specific to each group for 12 weeks. The authors reported that pelvic floor exercise was more effective in relieving PMD than bulbar urethral massage. On the other hand, counseling did not result in improvement. Also, the authors stated that bulbar urethral massage was effective immediately, whereas pelvic floor exercise needed 5 weeks to achieve a proper therapeutic effect. Dorey et al. [6] compared the efficacy of pelvic floor exercise and lifestyle modification on PMD in 36 males with PMD and ED. In males who received both pelvic floor exercise and lifestyle modification, $66.7 \%$ of patients at 3 months and $81.0 \%$ at 6 months reported no PMD. In males who received lifestyle modification, only $6.7 \%$ reported no PMD at 3 months. Also, they stated that bulbocavernosus muscle dysfunction might explain the association between $\mathrm{ED}$ and PMD.

Up-to-date, pharmacologic treatment for PMD has not yet been introduced. Recently, Ko et al. [8] assessed the effects of taking udenafil $75 \mathrm{mg}$, a PDE-5I, once daily, for 12 weeks, on PMD in 138 males with PMD and other LUTS. This randomized, double-blind, placebo-controlled trial first introduced the possibility of drug-based therapy for relieving PMD. They used the HPMDQ to assess the severity of PMD. They reported that PMD was improved in $61.7 \%$ of patients in the udenafil group at 12 weeks and in $26.9 \%$ of patients in the placebo group. Also, the reduction in PMD volume in the udenafil group was greater than in the placebo group. They suggested that taking udenafil $75 \mathrm{mg}$ once daily may be an effective treatment for PMD.

The mechanisms by which PDE-5I treats PMD are unknown. One putative mechanism is that PDE-5I may strengthen the urethro-corporocavernosal reflex. On urination, sinusoidal corpora muscle relaxation and cavernosa muscle contraction are suggested to be mediated through the urethro-corporocavernosal reflex [22]. These changes seem to cause a mild degree of penile tumescence and stretch, which can assist in urinary flow and may empty the residual urine out of the urethra [22-24]. Other putative mechanisms are associated with the corpus spongiosum and pelvic floor muscles. PDE-5I acts not only on the corpus cavernosum but also on the corpus spongiosum, to restore sinusoidal elasticity, which may correct dilated bulbar urethrae [8]. The pelvic floor muscles, especially the bulbocavernosus and ischiocavernosus muscles, contract to prevent blood from leaking during erections [35]. Like pelvic floor exercise, chronic treatment with a PDE-5I may help to strengthen the pelvic floor muscles.

\section{CONCLUSIONS}

PMD is the involuntary loss of urine immediately after urination. In contrast to what has traditionally been believed, it is not rare and does not elicit less discomfort compared with other LUTS. PMD often seems to overlap with other LUTS and to be associated with sexual function. Although the pathophysiology by which PMD occurs is not fully understood, a weakness or failure of pelvic floor 
muscles is considered to be the most important factor. Bulbar urethral massage and pelvic floor exercise are known to relieve PMD. Although pharmacologic treatment has not yet been introduced, the possibility of treatment with PDE-5Is has recently been suggested. Because available diagnostic tools and treatment options for PMD are lacking, PMD seems to be outside of physicians' interests. However, recent evidence suggests that PMD needs more attention. More research is needed to fully understand this disorder.

\section{CONFLICTS OF INTEREST}

The authors have nothing to disclose.

\section{SUPPLEMENTARY MATERIAL}

Scan this QR code to see the supplementary material, or visit https:/www.icurology.org/src/sm/icurology-60-142-s001.pdf.

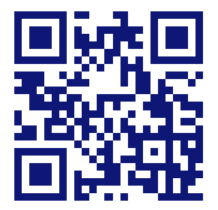

\section{REFERENCES}

1. Dorey G. Are erectile and ejaculatory dysfunction associated with postmicturition dribble? Urol Nurs 2003;23:42-5, 48-52.

2. De Nunzio C, Roehrborn CG, Andersson KE, McVary KT. Erectile dysfunction and lower urinary tract symptoms. Eur Urol Focus 2017;3:352-63.

3. Sexton CC, Coyne KS, Kopp ZS, Irwin DE, Milsom I, Aiyer LP, et al. The overlap of storage, voiding and postmicturition symptoms and implications for treatment seeking in the USA, UK and Sweden: EpiLUTS. BJU Int 2009;103 Suppl 3:12-23.

4. Irwin DE, Milsom I, Hunskaar S, Reilly K, Kopp Z, Herschorn $S$, et al. Population-based survey of urinary incontinence, overactive bladder, and other lower urinary tract symptoms in five countries: results of the EPIC study. Eur Urol 2006;50:1306-14; discussion 1314-5.

5. Yang DY, Ko K, Lee SH, Cho JS, Lee SK, Shin TY, et al. Postmicturition dribble is associated with erectile dysfunction in middle-aged and older men with lower uUrinary tract symptoms. World J Mens Health 2018;36:263-70.

6. Dorey G, Speakman M, Feneley R, Swinkels A, Dunn C, Ewings $P$. Pelvic floor exercises for treating post-micturition dribble in men with erectile dysfunction: a randomized controlled trial. Urol Nurs 2004;24:490-7, 512.

7. Dorey G. Prevalence, aetiology and treatment of post-micturi- tion dribble in men: literature review. Physiotherapy 2002;88: 225-34.

8. Ko K, Lee WK, Cho ST, Lee YG, Shin TY, Choo MS, et al. Effect of udenafil administration on postmicturition dribbling in men: a prospective, multicenter, double-blind, placebocontrolled, randomized clinical study. Aging Male 2019 Jan 16 [Epub]. https://doi.org/10.1080/13685538.2018.1545834.

9. Maserejian NN, Kupelian V, McVary KT, Doshi M, Link CL, McKinlay JB. Prevalence of post-micturition symptoms in association with lower urinary tract symptoms and health-related quality of life in men and women. BJU Int 2011;108:1452-8.

10. Pöyhönen A, Auvinen A, Koskimäki J, Hakama M, Tammela TL, Häkkinen JT. Prevalence and bother of postmicturition dribble in Finnish men aged 30-80 years: Tampere Ageing Male Urologic Study (TAMUS). Scand J Urol Nephrol 2012;46:41823.

11. Coyne KS, Sexton CC, Thompson CL, Milsom I, Irwin D, Kopp ZS, et al. The prevalence of lower urinary tract symptoms (LUTS) in the USA, the UK and Sweden: results from the Epidemiology of LUTS (EpiLUTS) study. BJU Int 2009;104:35260.

12. Agarwal A, Eryuzlu LN, Cartwright R, Thorlund K, Tammela TL, Guyatt GH, et al. What is the most bothersome lower urinary tract symptom? Individual- and population-level perspectives for both men and women. Eur Urol 2014;65:1211-7.

13. Pöyhönen A, Auvinen A, Häkkinen JT, Koskimäki J, Tammela TL. Population-level and individual-level bother of lower urinary tract symptoms among 30- to 80-year-old men. Urology 2016;95:164-70.

14. Abrams P, Cardozo L, Fall M, Griffiths D, Rosier P, Ulmsten U, et al.; Standardisation Sub-Committee of the International Continence Society. The standardisation of terminology in lower urinary tract function: report from the standardisation sub-committee of the International Continence Society. Urology 2003;61:37-49.

15. Hald T, Nordling J, Andersen JT, Bilde T, Meyhoff HH, Walter S. A patient weighted symptom score system in the evaluation of uncomplicated benign prostatic hyperplasia. Scand J Urol Nephrol Suppl 1991;138:59-62.

16. Wang $\mathrm{Y}, \mathrm{Hu} \mathrm{H}, \mathrm{Xu} \mathrm{K}$, Wang X, Na Y, Kang X. Prevalence, risk factors and the bother of lower urinary tract symptoms in China: a population-based survey. Int Urogynecol J 2015;26:911-9.

17. Ho LY, Chu PS, Consigliere DT, Zainuddin ZM, Bolong D, Chan CK, et al. Symptom prevalence, bother, and treatment satisfaction in men with lower urinary tract symptoms in Southeast Asia: a multinational, cross-sectional survey. World J Urol 2018;36:79-86.

18. Kobayashi K, Fukuta F, Masumori N. Prevalence of postmicturition dribble in Japanese men and its relationship with 
benign prostatic hyperplasia/lower urinary tract symptoms. Low Urin Tract Symptoms 2017 Dec 19 [Epub]. https://doi. org/10.1111/luts.12210.

19. Lee WC, Kim MT, Choo MS, Ko K, Shin TY, Jeong HB, et al. Study on post-micturition dribble in patients with lower urinary tract symptoms [abstract]. Annu Meet Korean Urol Assoc 2014. Abstract No. O-163.

20. Frankel SJ, Donovan JL, Peters TI, Abrams P, Dabhoiwala NF, Osawa D, et al. Sexual dysfunction in men with lower urinary tract symptoms. J Clin Epidemiol 1998;51:677-85.

21. Wein AJ, Coyne KS, Tubaro A, Sexton CC, Kopp ZS, Aiyer LP. The impact of lower urinary tract symptoms on male sexual health: EpiLUTS. BJU Int 2009;103 Suppl 3:33-41.

22. Shafik A, Shafik IA, El Sibai O, Shafik AA. Study of the response of the penile corporal tissue and cavernosus muscles to micturition. BMC Urol 2008;8:4.

23. Bleustein CB, Arezzo JC, Eckholdt H, Melman A. The neuropathy of erectile dysfunction. Int J Impot Res 2002;14:433-9.

24. Giuliano F, Rampin O. Central neural regulation of penile erection. Neurosci Biobehav Rev 2000;24:517-33.

25. Ferrer JE, Velez JD, Herrera AM. Age-related morphological changes in smooth muscle and collagen content in human corpus cavernosum. J Sex Med 2010;7:2723-8.

26. Costa C, Vendeira P. Does erectile tissue angioarchitecture modify with aging? An immunohistological and morphometric approach. J Sex Med 2008;5:833-40.
27. Stephenson TP, Farrar DJ. Urodynamic study of 15 patients with postmicturition dribble. Urology 1977;9:404-6.

28. Wille S, Mills RD, Studer UE. Absence of urethral post-void milking: an additional cause for incontinence after radical prostatectomy? Eur Urol 2000;37:665-9.

29. Bader P, Hugonnet CL, Burkhard FC, Studer UE. Inefficient urethral milking secondary to urethral dysfunction as an additional risk factor for incontinence after radical prostatectomy. J Urol 2001;166:2247-52.

30. Millard RJ. After-dribble. In: Millard RJ. Bladder control: a simple self-help guide. Sydney: Williams \& Wilkins; 1989;8990.

31. Pomfret IJ. Understanding post-micturition dribble incontinence in men. Nurs Times 2003;99:56-7.

32. Robinson J. Post-micturition dribble in men: causes and treatment. Nurs Stand 2008;22:43-6.

33. Bø K, Talseth T, Holme I. Single blind, randomised controlled trial of pelvic floor exercises, electrical stimulation, vaginal cones, and no treatment in management of genuine stress incontinence in women. BMJ 1999;318:487-93.

34. Paterson J, Pinnock CB, Marshall VR. Pelvic floor exercises as a treatment for post-micturition dribble. Br J Urol 1997;79:8927.

35. Wespes E, Nogueira MC, Herbaut AG, Caufriez M, Schulman CC. Role of the bulbocavernosus muscles on the mechanism of human erection. Eur Urol 1990;18:45-8. 
Supplementary material. The Hallym Post-Micturition Dribble Questionnaire (HPMDQ)

1. Over the last month, how often have you experienced dribbling after voiding when you feel you have finished urination?

0 . Not at all

1.1 out of 3 times

2. 2 out of 3 times

3. Almost always or always

1-(1). How much is dribbled urine after voiding?

1. Immediately after voiding, a little

2. Immediately after voiding, a lot

3. After wearing underwear, a little

4. After wearing underwear, a lot

2. Do you feel frustrated because of dribbling after voiding, when you feel you have finished urination?

0 . Not at all

1. Slightly

2. Moderately

3. A lot

3. If you were to spend the rest of your life with dribbling after voiding when you feel you have finished urination, how would you feel about that?

0 . Not dissatisfied

1. Slightly dissatisfied

2. Moderately dissatisfied

3. Very dissatisfied

4. Compared to before treatment, have you experienced improvement in dribbling after voiding when you feel you have finished urination?

0 . Not at all

1. Slightly

2. Moderately

3. A lot 